\title{
Low-Loss Matching Network Design for Band-Switchable Multi-Band Power Amplifier
}

\author{
Atsushi FUKUDA $^{\dagger \text { a) }}$, Takayuki FURUTA ${ }^{\dagger}$, Members, Hiroshi OKAZAKI ${ }^{\dagger}$, \\ Shoichi NARAHASHI ${ }^{\dagger}$, Senior Members, and Toshio NOJIMA ${ }^{\dagger \dagger}$, Fellow
}

\begin{abstract}
SUMMARY This paper presents a novel design scheme for a bandswitchable multi-band power amplifier (BS-MPA). A key point of the design scheme is configuring multi-section reconfigurable matching networks (MR-MNs) optimally in terms of low loss matching in multiple frequency bands from 0.7 to $2.5 \mathrm{GHz}$. The MR-MN consists of several matching sections, each of which has a matching block connected to a transmission line via a switch. Power dissipation at an actual on-state switch results in the insertion loss of the MR-MN and depends on how the impedance is transformed by the MR-MN. The proposed design scheme appropriately transforms the impedance of a high power transistor to configure a low loss MR-MN. Numerical analyses show quantitative improvement in the loss using the proposed scheme. A 9-band 3-stage BS-MPA is newly designed following the proposed scheme and fabricated on a multi-layer low temperature co-fired ceramic substrate for compactness. The BS-MPA achieves a gain of over $30 \mathrm{~dB}$, an output power of greater than $33 \mathrm{dBm}$ and a power added efficiency of over $40 \%$ at the supply voltage of $4 \mathrm{~V}$ in each operating band.

key words: power amplifier, reconfigurable, matching network, multiband, switch
\end{abstract}

\section{Introduction}

There has been an increase in the number of frequency bands allocated to mobile communication services provided all over the world. Future mobile terminals will be expected to function seamlessly in various mobile communication systems. Numerous research activities have been focused on adaptive RF circuits that can function according to any specification [1], [2].

The Power amplifier (PA) is a key device in RF circuits. The PA has a much greater impact on power dissipation than any other component in the RF circuits for mobile terminals. The PA must achieve a high level of operating efficiency in order to maximize the battery life time and minimize the size and cost of the terminals even if the PA has to cover a wide band or multiple bands. Several approaches have been developed for a multi-band PA [3]-[18]. A commonly used approach is "unit selection" [3], [4]. This scheme implements several PA units that operate efficiently in a single band or some adjacent bands. The circuit scale basically increases in proportion to the number of the PA

\footnotetext{
Manuscript received November 2, 2011.

Manuscript revised January 31, 2012.

${ }^{\dagger}$ The authors are with NTT DOCOMO, INC., Yokosuka-shi, 239-8536 Japan.

${ }^{\dagger \dagger}$ The author is with the Graduate School of Information Science and Technology, Hokkaido University, Sapporo-shi, 06008114 Japan.

a)E-mail: fukudaat@nttdocomo.co.jp

DOI: 10.1587/transele.E95.C.1172
}

units. Another approach is the "broadband matching" [5], [6] or "multi-band matching" [7]-[9] that covers all or several operating bands employing specially-designed matching networks (MNs). The former, which has a flat frequency response over all operating bands, is inappropriate to configure a highly efficient PA when the upper and lower limits of the operating bands are far apart. The latter, which achieves optimum matching simultaneously in multiple bands, needs complex circuits to provide multi-band operation. Moreover, designing multi-band MNs becomes difficult when the number of the operating bands increases. One practical approach for the multi-band PA is to employ "reconfigurable MNs" [10]-[18] that change their frequency responses using variable devices. This type of structure represents a promising solution because it has a reasonably sized circuit configuration considering multiple band operation while providing adequate characteristics.

There have been reports on a 1-W class band reconfigurable PA employing micro-electro-mechanical systems (MEMS) Single-Pole-Single-Throw (SPST) switches [14][16] as variable devices. The PA includes multi-section reconfigurable MNs (MR-MNs), which basically have one switch in one section as shown in Fig. 1(a), and exhibits performance levels close to those of a single-band PA in the 1, 1.5, 1.9 and 2.5-GHz bands [16]. The performance of the MEMS switch is close to that of an ideal switch with both a low insertion loss and a high isolation. However, there are some issues that need to be addressed in applying MEMS switches to mobile terminals, such as cost reduction, ensuring long-term reliability, and reducing the operating voltage. From a more practical view, applying semiconductor switches instead of MEMS switches is attractive to not only solve the above-mentioned issues but also facilitate a high level of integration for the PA. A bandswitchable multi-band power amplifier (BS-MPA) employing GaAs FET switches was fabricated and tested [17], [18].

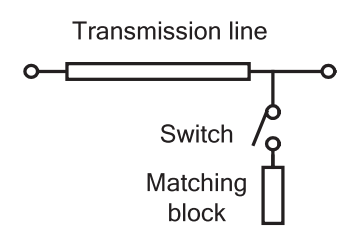

(a) One switch in one section

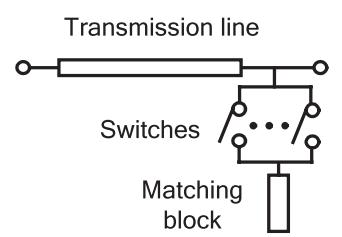

(b) Several switches in one section
Fig. 1 Configurations of one section in the MR-MN 
The fabricated BS-MPA achieves an adequate gain and an output power of over several watts in 9 bands from 0.7 to $2.5 \mathrm{GHz}$ allocated to mobile communications all over the world. However, since the equivalent resistance of the onstate FET switch is higher than that of the MEMS switch used in the previous studies, the power dissipation at the FET switch has a stronger influence on the performance of the PA than that from the MEMS switch. In [17] and [18], as shown in Fig. 1(b), some sections employed several switches instead of one switch in one section to reduce the current flowing through each on-state switch and the power dissipation at the FET switch. Reduction in the circuit size and cost of the configuration were required because many switches were implemented.

This paper presents a novel design scheme to configure the MR-MN with lower power dissipation at the onstate switch for low loss matching in individual bands. The scheme is based on the use of several sections that have one switch. This paper also describes numerical quantitative analyses of the power dissipations at the on-state switches based on the relational expressions between the impedance transformation and the current flowing through the switch. These analysis results yield design parameters to achieve the low loss MR-MN and indicate improvement in the insertion loss by the MR-MN with the determined parameters compared to the conventional MR-MN. Following the design parameters for the MR-MN, a 3-stage 9-band BS-MPA from $0.7 \mathrm{GHz}$ to $2.5 \mathrm{GHz}$, i.e., 0.7, 0.8, 0.9, 1.5, 1.7, 1.8, 1.9, 2.3 , and $2.5 \mathrm{GHz}$ bands, is newly designed and fabricated on a multi-layer Low Temperature Co-fired Ceramic (LTCC) substrate. The experimental results of the BS-MPA show a gain exceeding $30 \mathrm{~dB}$ for each frequency band. The BSMPA achieves an output power exceeding $33 \mathrm{dBm}$ with the maximum power-added efficiency (PAE) of over $40 \%$ with a continuous wave $(\mathrm{CW})$ test signal in the targeted 9 bands. In addition, an output power of approximately $29 \mathrm{dBm}$ under an Adjacent Channel Leakage Ratio (ACLR) of less than $-37 \mathrm{dBc}$ is achieved with a W-CDMA test signal.

\section{Band-Switchable Multi-Band PA}

Figure 2 shows a block diagram of the 3-stage BS-MPA. Since the output power of the first stage is at a low or medium level, broadband amplifiers with moderate efficiency can be applied to this stage. The second and third stages have a greater effect on the power consumption than the first stage. Therefore, the MNs for these two stages should be configured with the MR-MNs to achieve matching precisely.

\subsection{Basic Configuration for MR-MN}

The MR-MNs proposed in [14] provide practical configurations for a reconfigurable MN. Figure 3 shows the basic configuration as the output $\mathrm{MN}$ for $\mathrm{N}$-band operation. Here, $B_{n}(1 \leq n \leq N)$ is the $n$-th band with the center frequency of $f_{n}$. The $f_{n}$ is allocated in accordance with $f_{1}>\cdots>f_{N}$.

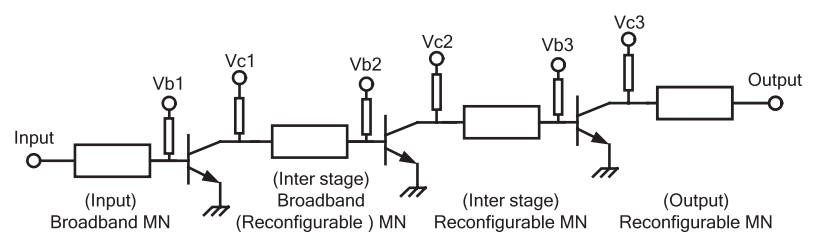

Fig. 2 Block diagram of a 3-stage power amplifier.

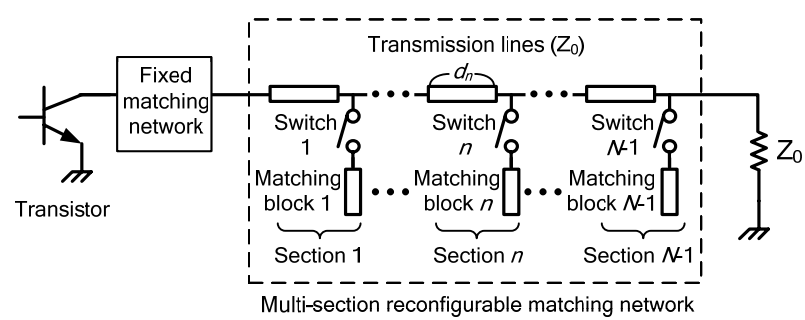

Fig. 3 Basic configuration of a multi-section reconfigurable matching network applied to output matching network.

The whole MN includes a fixed MN and a MR-MN that consists of $\mathrm{N}-1$ sections. One section has a shunt SPST switch connected to a transmission line (TL) for reconfiguration. In Fig. 3, the length of the TL, $d_{n}$, and the reactance of matching block $n$ in the section $n$ are designed to achieve matching in $B_{n+1}$ on the assumption that switch $n$ is in the on-state in single stub matching scheme [19]. The characteristic impedance of the TL is set to $\mathrm{ZO}$ because the following sections prevent the impedances in the matched bands from being unmatched. The fixed $\mathrm{MN}$ is designed for matching in $B_{1}$. The configurations of the fixed $\mathrm{MN}$ and each section basically have low-pass characteristics. The MR-MNs are reconfigured in accordance with the change in the operating band and provide an amplification device with the optimum impedance condition in each of multiple bands. Since no switches are inserted in series into the output signal paths, the configuration is expected to have less effect on the output power (Pout) and PAE. The same configuration can be applied to the input MN as well.

\subsection{Low Loss Design for MR-MN}

A simple equivalent circuit for an actual switch is described as a resistor for the low impedance in the on-state. Particularly, the power dissipation at the on-state switch becomes potentially a serious issue due to decreases in the output power and operating efficiency. Figure 4 shows a circuit diagram for investigating the power dissipation at the onstate switch employed for matching in $B_{n+1}$ in Fig. 3. All switches except switch $n$ are in the off-state. The off-state switches are not shown in Fig. 4 because their isolation is assumed to be infinite for the sake of simplicity. In Fig. 4, the TL length is the sum of the TLs between the first section and the section $n$ in Fig. 3. In Fig. $4, Z_{\mathrm{T}}$ is the output impedance of the transistor. Term $Z_{\mathrm{g}}$ is the impedance where $Z_{\mathrm{T}}$ is transformed by the fixed $\mathrm{MN}$.

Power dissipation, $P_{\mathrm{sw} 1}$, at the on-state switch, can be 


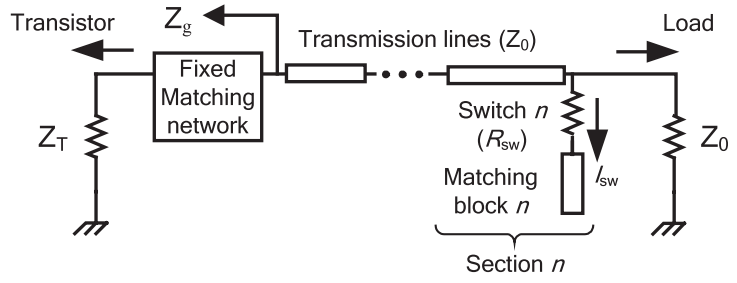

Fig. 4 Circuit diagram for investigating power dissipation at on-state switch.

written as

$$
P_{\mathrm{sw} 1}=I_{\mathrm{sw}}^{2} \cdot R_{\mathrm{sw}},
$$

where $R_{\mathrm{sw}}$ is the equivalent resistance of the on-state switch and $I_{\mathrm{sw}}$ is the root mean square (RMS) RF current flowing through the switch. Here, $I_{\mathrm{sw}}$ is given as

$$
I_{\mathrm{sw}}=\sqrt{\frac{P}{Z_{0}}} \sqrt{\frac{4\left|\Gamma_{\mathrm{g}}\right|^{2}}{1-\left|\Gamma_{\mathrm{g}}\right|^{2}}},
$$

where the $P$ is the power delivered to the load, $Z_{0},[15]$ and a mismatch loss by $R_{\mathrm{sw}}$ is not taken into account. The $\left|\Gamma_{\mathrm{g}}\right|$ is the magnitude of the complex $\Gamma_{\mathrm{g}}$, which is the relation between $Z_{\mathrm{g}}$ and $Z_{0}$, and defined as

$$
\Gamma_{\mathrm{g}}=\frac{Z_{\mathrm{g}}-Z_{0}}{Z_{\mathrm{g}}+Z_{0}}=\left|\Gamma_{\mathrm{g}}\right| e^{j \theta_{\mathrm{g}}},
$$

Then, the insertion loss of the MR-MN for matching in $B_{n+1}$, $L_{n 1}$, can be estimated as

$$
L_{n 1}=P /\left(P-P_{\mathrm{sw} 1}\right)=1 /\left[1-\frac{R_{\mathrm{sw}}}{Z_{0}}\left(\frac{4\left|\Gamma_{\mathrm{g}}\right|^{2}}{1-\left|\Gamma_{\mathrm{g}}\right|^{2}}\right)\right] .
$$

From Eq. (2), $I_{\mathrm{sw}}$ monotonically increases with respect to the increase in $\left|\Gamma_{\mathrm{g}}\right|$. Then, $P_{\mathrm{sw} 1}$ and $L_{n 1}$ also increase as $\left|\Gamma_{\mathrm{g}}\right|$ increases as in Eqs. (1) and (4), respectively. For example, if $P=1 \mathrm{~W},\left|\Gamma_{\mathrm{g}}\right|=0.9$ and $R_{\mathrm{sw}}=1 \Omega, L_{n 1}=1.81 \mathrm{~dB}$ is obtained from Eq. (4). On the other hand, the value for $\left|\Gamma_{\mathrm{g}}\right|$ in each band is determined by $Z_{\mathrm{T}}$ and the fixed MN. Namely, the fixed $\mathrm{MN}$ is employed not only to configure the $\mathrm{MN}$ for $B_{1}$ but also to reduce the power dissipation at the switches included in the MR-MN. Here, $Z_{\mathrm{T}}$ is assumed to be constant in terms of frequency because the $Z_{\mathrm{T}}$ values of the actual transistor are approximately constant in the target bands. When $Z_{\mathrm{T}}$ is satisfied by $\left|\Gamma_{\mathrm{T}}\right|=0.9$ and $\theta_{\mathrm{T}}=180 \mathrm{de}-$ grees from Eq. (3), the calculated $\left|\Gamma_{\mathrm{g}}\right|, L_{n 1}$, and the improvement in the loss compared to that for the MR-MN without the fixed MN are indicated in Table 1. Here, 9 bands allocated for mobile communications are selected as the target bands for calculation. It is assumed that the TL and matching blocks are lossless. Table 1 shows that the $L_{n 1} \mathrm{~s}$ in the higher bands are less than those in the lower bands because the $\left|\Gamma_{\mathrm{g}}\right|$ values in the higher bands become smaller than those in the lower bands due to the fixed MN. There is no loss from the switch for matching in the $2.5-\mathrm{GHz}$ band. The improvements in the insertion loss are verified to be more than $1 \mathrm{~dB}$
Table 1 Insertion loss and improvement effect for fixed MN

\begin{tabular}{|c|c|c|c|c|c|c|c|c|c|}
\hline $\begin{array}{c}\text { Frequency } \\
(\mathrm{GHz})\end{array}$ & 0.7 & 0.8 & 0.9 & 1.5 & 1.7 & 1.8 & 1.9 & 2.3 & 2.5 \\
\hline$\left|\Gamma_{\mathrm{g}}\right|$ & 0.89 & 0.88 & 0.87 & 0.80 & 0.74 & 0.70 & 0.66 & 0.30 & 0 \\
\hline$\theta_{\mathrm{g}}(\mathrm{deg})$. & 173 & 171 & 170 & 159 & 154 & 150 & 146 & 121 & 0 \\
\hline$L_{n 1}(\mathrm{~dB})$ & 1.58 & 1.39 & 1.24 & 0.67 & 0.44 & 0.35 & 0.28 & 0.03 & 0 \\
\hline $\begin{array}{c}\text { Improvement } \\
(\mathrm{dB})\end{array}$ & 0.23 & 0.42 & 0.57 & 1.14 & 1.37 & 1.46 & 1.53 & 1.78 & 0 \\
\hline
\end{tabular}

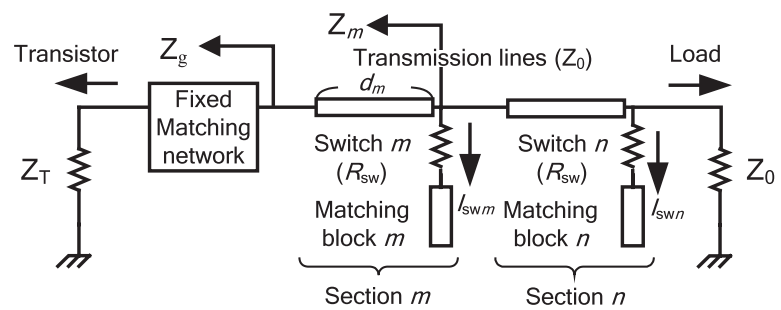

Fig. 5 Circuit diagram for investigating the insertion loss due to two sections with on-state switches.

in the $1.5-\mathrm{GHz}$ and higher bands. In general, the low loss characteristics of the $\mathrm{MN}$ in the high bands have additional advantages because it is more difficult to obtain an adequate gain or efficiency level depending on the performance of the transistor as the operating bands become higher.

For further improvement in the lower bands, the newly proposed design scheme employs several sections simultaneously to decrease the power dissipation at the on-state switch. In this case, the insertion loss of the MR-MN is the sum of the power dissipation at each switch in the onstate. As the simplest case, Fig. 5 shows a circuit diagram for investigating the insertion loss of the MR-MN with two sections including the on-state switch, namely section $m$ and section $n$. Section $m$ is the newly added section or any section between section 1 and section $n-1$. The values of the currents flowing through the on-state switches are described as $I_{\text {sw } m}$ and $I_{\text {sw } n}$ in Fig. 5. In Fig. 5, $Z_{\mathrm{g}}$ is transformed to $Z_{m}$ by section $m$ and then $Z_{m}$ is transformed to $Z_{0}$ by section $n$.

The relation, $\Gamma_{m}$, between $Z_{0}$ and $Z_{m}$ in Fig. 5 is defined as

$$
\Gamma_{m}=\frac{Z_{m}-Z_{0}}{Z_{m}+Z_{0}}=\left|\Gamma_{m}\right| e^{j \theta_{m}}
$$

where $\left|\Gamma_{m}\right|$ and $\theta_{m}$ are the magnitude and phase angle of complex $\Gamma_{m}$, respectively. Then, from Eq. (5),

$$
G_{m}=Y_{0} \frac{1-\left|\Gamma_{m}\right|^{2}}{1+2\left|\Gamma_{m}\right| \cos \theta_{m}+\left|\Gamma_{m}\right|^{2}}
$$

and

$$
B_{m}=Y_{0} \frac{-2\left|\Gamma_{m}\right| \sin \theta_{m}}{1+2\left|\Gamma_{m}\right| \cos \theta_{m}+\left|\Gamma_{m}\right|^{2}},
$$

where $Y_{m}=1 / Z_{m}=G_{m}+j B_{m}$ and $Y_{0}=1 / Z_{0}$. On the other hand, in Fig. 5, the reactance, $Y\left(d_{m}\right)=G\left(d_{m}\right)+j B\left(d_{m}\right)$, at the distance of electrical length, $d_{m}$, from the fixed $\mathrm{MN}$ on the TL is given as 


$$
G\left(d_{m}\right)=Y_{0} \frac{1-\left|\Gamma_{\mathrm{g}}\right|^{2}}{1+2\left|\Gamma_{\mathrm{g}}\right| \cos \left(2 d_{m}-\theta_{\mathrm{g}}\right)+\left|\Gamma_{\mathrm{g}}\right|^{2}}
$$

and

$$
B\left(d_{m}\right)=Y_{0} \frac{2\left|\Gamma_{\mathrm{g}}\right| \sin \left(2 d_{m}-\theta_{\mathrm{g}}\right)}{1+2\left|\Gamma_{\mathrm{g}}\right| \cos \left(2 d_{m}-\theta_{\mathrm{g}}\right)+\left|\Gamma_{\mathrm{g}}\right|^{2}} .
$$

Since $G_{m}=G\left(d_{m}\right)$ from Eqs. (6) and (8), we have

$$
\cos \left(2 d_{m}-\theta_{\mathrm{g}}\right)=\frac{\left|\Gamma_{m}\right|^{2}-\left|\Gamma_{\mathrm{g}}\right|^{2}+\left|\Gamma_{m}\right|\left(1-\left|\Gamma_{\mathrm{g}}\right|^{2}\right) \cos \theta_{m}}{\left|\Gamma_{\mathrm{g}}\right|\left(1-\left|\Gamma_{m}\right|^{2}\right)} .
$$

From Eq. (10), $d_{m}$ can be written as

$$
d_{m}=\frac{1}{2}\left(\cos ^{-1} \alpha+\theta_{\mathrm{g}}\right) \text {, }
$$

where $\alpha$ is the right member of Eq. (10). When $\pi / 2 \leq$ $\cos ^{-1} \alpha \leq \pi$, the minimum $d_{m}$ depends on $\theta_{\mathrm{g}}$ and is given as

$$
d_{m}=\left\{\begin{array}{ll}
\frac{1}{2}\left\{2 \pi-\cos ^{-1}(\alpha)+\theta_{\mathrm{g}}\right\} & \text { at }-\pi<\theta_{\mathrm{g}}<-\cos ^{-1}(\alpha) \\
\frac{1}{2}\left\{\cos ^{-1}(\alpha)+\theta_{\mathrm{g}}\right\} & \text { at } 0 \leq\left|\theta_{\mathrm{g}}\right| \leq \cos ^{-1}(\alpha) \\
\frac{1}{2}\left\{-\cos ^{-1}(\alpha)+\theta_{\mathrm{g}}\right\} & \text { at } \cos ^{-1}(\alpha)<\theta_{\mathrm{g}}<\pi
\end{array} .\right.
$$

Then the susceptance of matching block $m, B_{\mathrm{b} m}$, can be written as

$$
B_{\mathrm{b} m}=B_{m}-B\left(d_{m}\right) .
$$

At the matching condition, the RMS voltage, $V_{d m}$, at $d_{m}$ from the fixed MN in Fig. 5 can be written as

$$
V_{d m}=\sqrt{P / G_{m}} .
$$

From Eqs. (13) and (14), $I_{\mathrm{sw} m}$ is given as

$$
I_{\mathrm{sw} m}=\left|B_{\mathrm{b} m}\right| \sqrt{P Z_{0}} \sqrt{\frac{1+2\left|\Gamma_{m}\right| \cos \theta_{m}+\left|\Gamma_{m}\right|^{2}}{1-\left|\Gamma_{m}\right|^{2}}} .
$$

Here, when Eq. (15) is derived, the mismatch loss by $R_{\mathrm{sw}}$ is not taken into account. Figure 6 shows plots of $I_{\mathrm{sw} m}$ as a function of $\theta_{m}$. Here, parameters of $P=1 \mathrm{~W},\left|\Gamma_{\mathrm{g}}\right|=0.9$, and $\theta_{\mathrm{g}}=180$ degrees are selected as an example. According to Fig. 6, $I_{\mathrm{sw} m}$ depends on $\Gamma_{m}$ transformed from $\Gamma_{\mathrm{g}}$ by section $m$. Meanwhile, $I_{\mathrm{sw} n}$ can be calculated from Eq. (2) by substituting $\left|\Gamma_{m}\right|$ instead of $\left|\Gamma_{\mathrm{g}}\right|$ after $\left|\Gamma_{m}\right|$ is determined.

The total power dissipation, $P_{\mathrm{sw} 2}$, due to two switches can be written as

$$
P_{\mathrm{sw} 2}=I_{\mathrm{sw} m}^{2} \cdot R_{\mathrm{sw}}+I_{\mathrm{sw} n}^{2} \cdot R_{\mathrm{sw}} .
$$

Then $L_{n 2}$, which is the insertion loss of the MR-MN for matching in $B_{n+1}$, can be expressed as

$$
L_{n 2}=\frac{1}{1-R_{\mathrm{sw}}\left\{\left|B_{\mathrm{b} m}\right|^{2} Z_{0}\left(\frac{1+2\left|\Gamma_{m}\right| \cos \theta_{m}+\left.\Gamma_{m}\right|^{2}}{1-\left.\Gamma_{m}\right|^{2}}\right)+\frac{1}{Z_{0}}\left(\frac{4\left|\Gamma_{m}\right|^{2}}{1-\left.\Gamma_{m}\right|^{2}}\right)\right\}}
$$

Figure 7 shows plots of $L_{n 2}$ as a function of $\theta_{m}$. Parameters

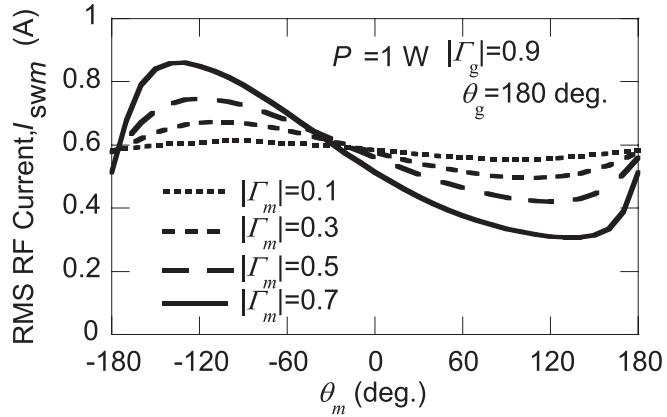

Fig. 6 Root mean square (RMS) RF current flowing through the switch

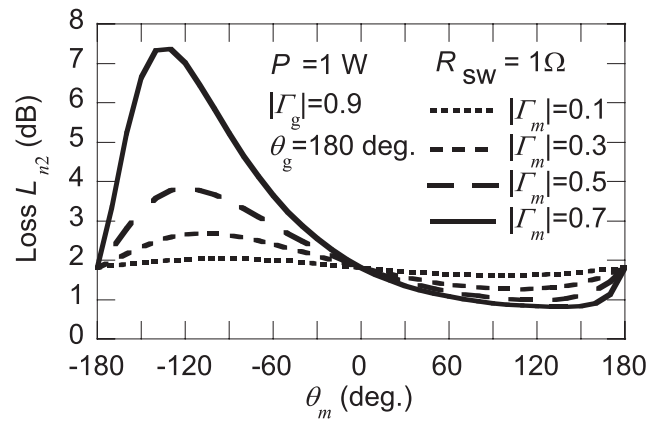

Fig. 7 Estimated insertion loss of the MR-MN due to two sections with the on-state switches as a function of $\theta_{m}$.

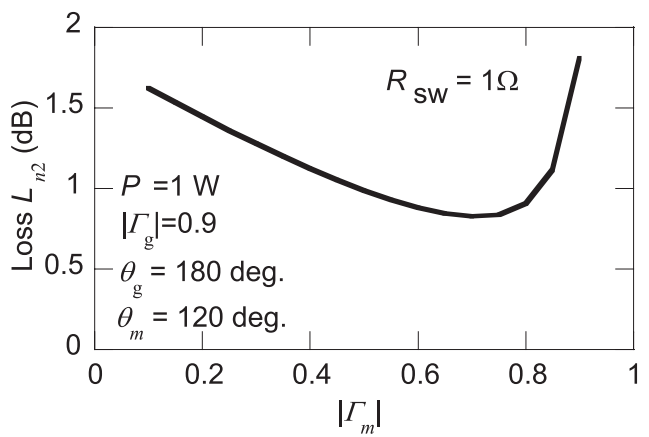

Fig. 8 Estimated insertion loss of the MR-MN due to two sections with the on-state switches as a function of $\left|\Gamma_{m}\right|$.

of $P=1 \mathrm{~W},\left|\Gamma_{\mathrm{g}}\right|=0.9, \theta_{\mathrm{g}}=180$ degrees, and $R_{\mathrm{sw}}=1 \Omega$ are selected as an example. Loss $L_{n 2}$ has the lowest value around $\theta_{m}=120$ degrees at each $\left|\Gamma_{m}\right|$. Figure 8 shows plots of $L_{n 2}$ as a function of $\left|\Gamma_{m}\right|$ at $\theta_{m}=120$ degrees and $L_{n 2}$ has the lowest value around $\left|\Gamma_{m}\right|=0.7$. The low $\left|\Gamma_{m}\right|$ value means a large impedance transformation between $Z_{\mathrm{g}}$ and $Z_{m}$ by section $m$, and as a result, $L_{n 2}$ becomes large due to the large $I_{\mathrm{sw} m}$. On the other hand, a $\left|\Gamma_{m}\right|$ value greater than 0.8 means a large impedance transformation between $Z_{m}$ and $Z_{0}$ by section $n$, and $L_{n 2}$ becomes large due to large $I_{\mathrm{sw} n}$. As shown in Figs. 7 and 8, $L_{n 2}$ can be decreased below $1 \mathrm{~dB}$, nevertheless, $\left|\Gamma_{\mathrm{g}}\right|=0.9$ when the loss by one section, $L_{n 1}$, is $1.81 \mathrm{~dB}$, which is obtained from Eq. (4).

Table 2 shows a summary of the calculation results on the impedance transformations and the total improvement 
Table 2 Insertion loss and total improvement for the MR-MN.

\begin{tabular}{|c|c|c|c|c|c|c|c|c|c|}
\hline $\begin{array}{c}\text { Frequency } \\
(\mathrm{GHz})\end{array}$ & 0.7 & 0.8 & 0.9 & 1.5 & 1.7 & 1.8 & 1.9 & 2.3 & 2.5 \\
\hline$\left|\Gamma_{\mathrm{g}}\right|$ & 0.89 & 0.88 & 0.87 & 0.80 & 0.74 & 0.70 & 0.66 & 0.30 & 0 \\
\hline$\theta_{\mathrm{g}}$ (deg.) & 173 & 171 & 170 & 159 & 154 & 150 & 146 & 121 & 0 \\
\hline$\left|\Gamma_{m}\right|$ & \multicolumn{4}{|c|}{0.7} & \multirow{2}{*}{ N/A } & \multirow{2}{*}{ N/A } & \multirow{2}{*}{ N/A } & \multirow{2}{*}{ N/A } & \multirow{2}{*}{ N/A } \\
\hline$\theta_{m}$ (deg.) & \multicolumn{4}{|c|}{120} & & & & & \\
\hline$L_{n 2}(\mathrm{~dB})$ & 0.73 & 0.65 & 0.59 & 0.4 & 0.44 & 0.35 & 0.28 & 0.03 & 0 \\
\hline $\begin{array}{c}\text { Total } \\
\text { improvement } \\
\text { (dB) }\end{array}$ & 1.08 & 1.16 & 1.22 & 1.41 & 1.37 & 1.46 & 1.53 & 1.78 & 0 \\
\hline
\end{tabular}

in the insertion loss due to the one or two sections. Here, the MR-MN due to the two sections is applied in the bands lower than $1.7-\mathrm{GHz}$ band because of the insertion loss that exceeds $0.5 \mathrm{~dB}$ in the one section from Table 1 . Improvement of more than $1 \mathrm{~dB}$ in the insertion loss is obtained by designing matching block $m$ so that it is satisfied by $\left|\Gamma_{m}\right|=$ 0.7 and $\theta_{m}=120$ degrees in the bands lower than $1.7-\mathrm{GHz}$ band. In a practical design, any one section between the first section and the section $n-1$ can be applied as section $m$ to achieve the condition regarding $\left|\Gamma_{m}\right|$ and $\theta_{m}$ instead of adding a new section. It is also possible to employ more than two sections for a lower loss MR-MN using the existing sections. This approach is very effective to reduce the circuit size and cost.

\section{Design of MR-MN with FET Switches}

To demonstrate the validity of the proposed design scheme, a 9-band PA was designed considering the characteristics of FET switches. The PA has 3 stages to provide the total gain of over $30 \mathrm{~dB}$. Each stage is designed individually to match $50 \Omega$ based on prior testing in each stage. The third stage is designed to achieve power matching based on nonlinear simulations. In the design, the MR-MNs are adopted in not only the third stage but also the second stage. InGaP heterojunction bipolar transistors (HBTs) are used as amplification devices. Nine bands, 0.7, 0.8, 0.9, 1.5, 1.7, 1.8, $1.9,2.3$, and $2.5 \mathrm{GHz}$, are selected as the applied or applicable bands for mobile communications all over the world. Figure 9 shows the measured insertion loss and isolation of the switch. Here, the effect of bonding wires and feed TL loss are included. The switch has an insertion loss of less than $0.7 \mathrm{~dB}$ and an isolation of greater than $13 \mathrm{~dB}$ over a frequency band below $3 \mathrm{GHz}$.

The power handling capability of the switch is also tested at $2.5 \mathrm{GHz}$ as a representative frequency and shown in Fig. 10. The insertion loss and the isolation are almost constant at the input power of 15 to approximately $35 \mathrm{dBm}$.

Figure 11 shows the third-order intermodulation distortion (IMD3) characteristics of the switch at various total power levels for 2-tone $\mathrm{CW}$ input signals $\left(f_{1}=2.500 \mathrm{GHz}\right.$, $f_{2}=2.501 \mathrm{GHz}$ ). The third order input intercept point (IIP3) is estimated to be $67 \mathrm{dBm}$.

The power handling capability and distortion characteristics of the employed FET switch are thought to be satis-

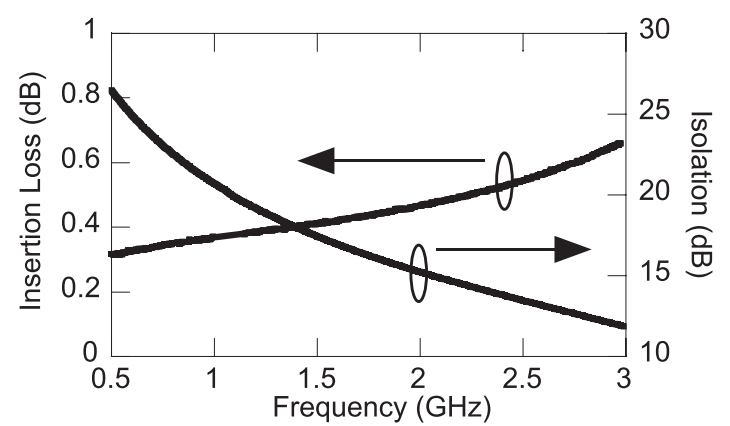

Fig. 9 Measured insertion loss and isolation of the employed FET switch.

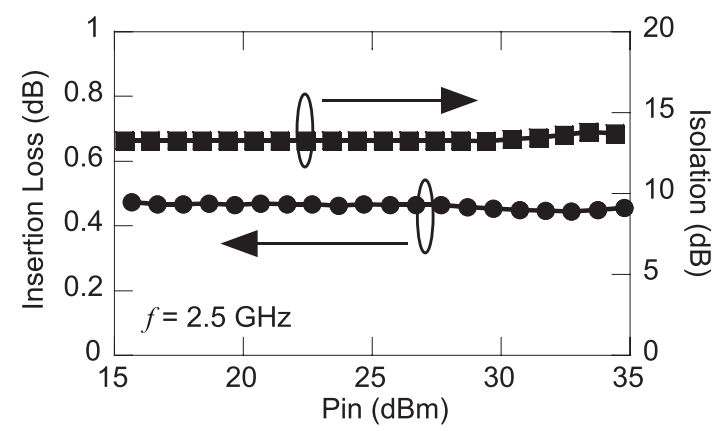

Fig. 10 Measured power handling capabilities of the employed FET switch.

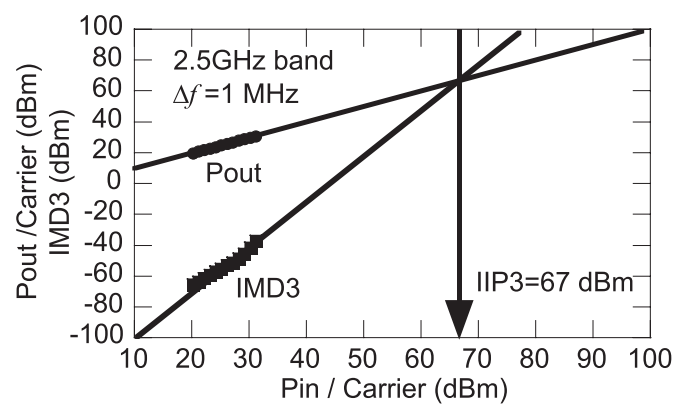

Fig. 11 Measured third order intermodulation distortion (IMD3) characteristics of the employed FET switch.

factory to be applied to the MR-MN because the FET switch is a commercially available antenna switch for GSM application. However, the power dissipation at the FET switch, which is higher than that of the MEMS switch employed in previous reports, has a great impact on the output power and PAE degradations of the high power BS-MPA. In the design of the 9-band PA, the configuration following the proposed design scheme is adopted in the MR-MN in the third stage where the power dissipation at the switches is outside the allowable range. Figures 12(a), (b), and (c) show the schematics of the first, second, and third stages of the newly designed 9-band 3-stage BS-MPA, respectively. The first stage with broadband MNs operates in a wide frequency band. According to the basic configuration of the MR-MN shown in Figs. 3, 16 sections ( 8 for input and 8 for output $\mathrm{MNs}$ ) in each stage are required for matching in the 9 bands. 


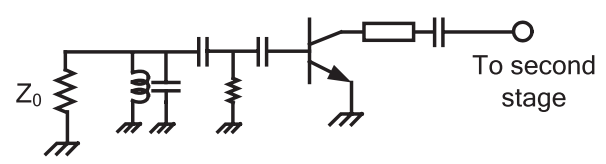

(a) First stage
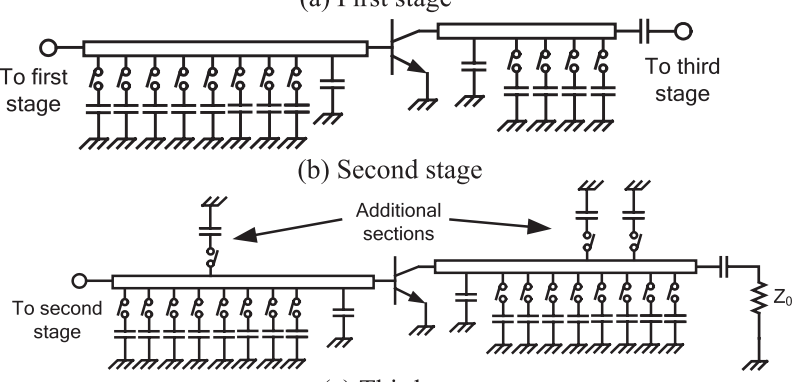

(c) Third stage

Fig. 12 Schematics of the newly designed 9-band 3-stage BS-MPA.

However, 12 sections ( 8 for input and 4 for output MNs) are required for the second stage. Four sections suffice as the output $\mathrm{MN}$ because several sections cover plural bands. On the other hand, the third stage has 19 sections because three sections are added by the low loss design scheme described in Sect. 2.2. The total number of switches are 31.

\section{Fabrication and Measurement}

\subsection{Fabrication}

A prototype of the 9-band 3-stage BS-MPA was fabricated using LTCC technology. The dielectric constant and total thickness of the substrate are 7.1 and $0.5 \mathrm{~mm}$, respectively. RF signal lines, active devices (HBTs and switches), and discrete passive devices are implemented on the top layer. Because many bias lines for active devices can be implemented in the intermediate layers by employing the multi-layer substrate, compact integration of the BS-MPA can be achieved. A photograph of the fabricated BS-MPA is shown in Fig. 13. The size of the LTCC substrate is $25 \mathrm{~mm} \times 25 \mathrm{~mm}$. A middle-power HBT with the emitter size of $1,248 \mu \mathrm{m}^{2}$ and 2 high-power HBTs with the emitter size of $7,488 \mu \mathrm{m}^{2}$ are deployed on the substrate for the first stage and for the second and third stages, respectively. In Fig. 13, there are over 31 discrete switches and lumped elements because some of switches and elements are used for adjustment for matching in the experiment.

\subsection{Measurement}

Figure 14 shows the measured frequency responses of the fabricated BS-MPA at several switch states for the representative operation modes: (a) $0.7-\mathrm{GHz}$, (b) $0.8-\mathrm{GHz}$, (c) 0.9-GHz, (d) $1.5-\mathrm{GHz}$, (e) $1.7-\mathrm{GHz}$, (f) $1.8-\mathrm{GHz}$, (g) $1.9-$ $\mathrm{GHz}$, (h) 2.3-GHz, and (i) 2.5-GHz modes. In Fig. 14, the 9-band BS-MPA achieves a gain of over $30 \mathrm{~dB}$ around each center frequency. Because of the influences of the bonding wires, the center frequencies in modes (h) and (i) shift lower by $50 \mathrm{MHz}$ and $100 \mathrm{MHz}$, respectively. The collector supply

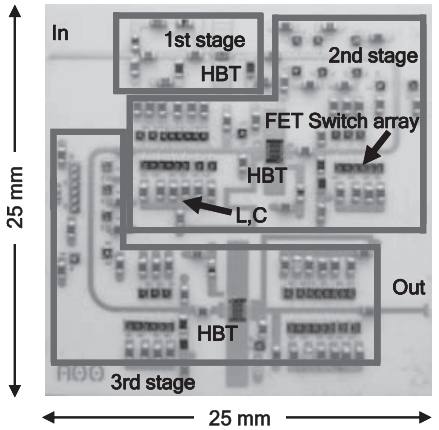

Fig. 13 Photograph of the fabricated 9-band 3-stage BS-MPA.

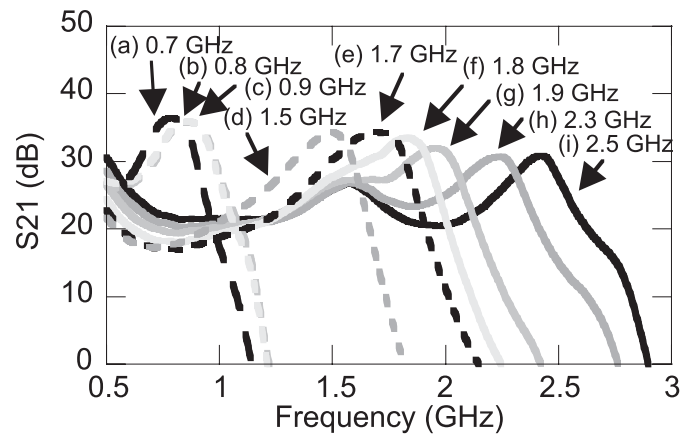

Fig. 14 Measured frequency responses of the fabricated BS-MPA.

voltage $\left(V_{c}\right)$ and base supply voltage $\left(V_{\mathrm{b}}\right)$ are set to $3.5 \mathrm{~V}$ and $1.3 \mathrm{~V}$ (Class-A) for the first stage, $3.5 \mathrm{~V}$ and $1.28 \mathrm{~V}$ (Class$\mathrm{AB}$ ) for the second stage, and $4 \mathrm{~V}$ and $1.26 \mathrm{~V}$ (Class-B) for the third stage, respectively. The results show that the BSMPA successfully changes its frequency response through the activation of FET switches. We also verified that it can achieve a level of performance close to that for band-free operation. No undesired oscillations are observed in any of the modes.

Figure 15 shows the Pout and PAE with the CW test at the center frequency of each mode for the (a) low band modes of $0.7 / 0.8 / 0.9 \mathrm{GHz}$, (b) middle band modes of 1.5/1.7/1.8/1.9 GHz, and (c) high band modes of 2.3/2.5 GHz. The HBTs are biased under the same conditions described above. According to Fig. 15, the gain expansion in a high output power range is observed in each frequency.

Table 3 shows a summary of the measurement results for the $\mathrm{CW}$ test. The gains in the low band modes are compressed by the first stage so that the difference in gain is small among operating bands. As a result, the difference in gain among the 9 bands is $5.6 \mathrm{~dB}$. More specifically, the maximum gain of $36.4 \mathrm{~dB}$ in mode (a) and the minimum gain of $30.8 \mathrm{~dB}$ in mode (i) are achieved because of the intrinsic gain of the HBTs. The saturation power level (Psat) exceeds $33 \mathrm{dBm}$ in all bands. The maximum PAE value for each mode is over $40 \%$. The Psat and maximum PAE at $50 \mathrm{MHz}$ around the center frequency in each mode are also indicated to be over $33 \mathrm{dBm}$ and $38 \%$, respectively. It is verified that the PA operates with an adequate bandwidth in 


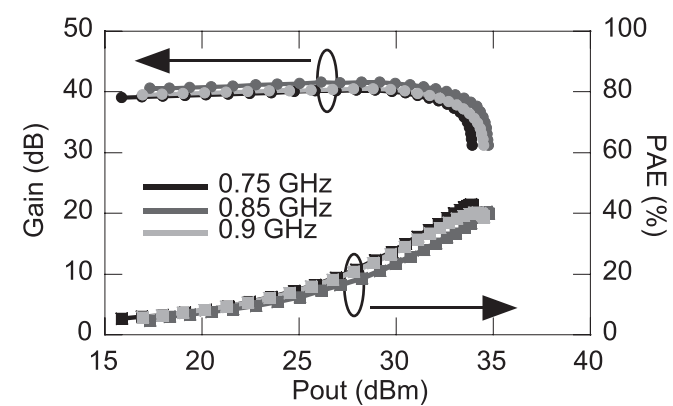

(a) Low band

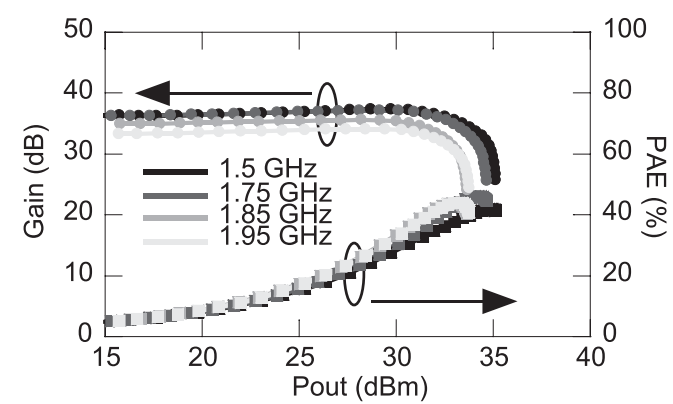

(b) Middle band

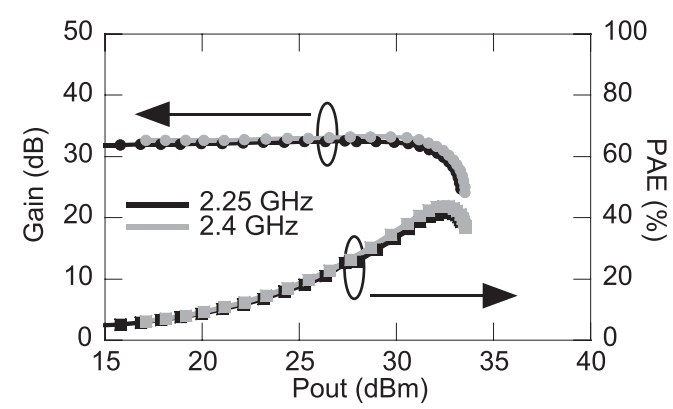

(c) High band

Fig. 15 Measured gain and PAE versus Pout at the center frequency of each mode.

Table 3 Measurement results on CW test.

\begin{tabular}{|c|c|c|c|c|c|c|c|c|c|}
\hline Mode & (a) & (b) & (c) & (d) & (e) & (f) & (g) & (h) & (i) \\
\hline $\begin{array}{c}\text { Frequency } \\
\text { (GHz) }\end{array}$ & 0.75 & 0.85 & 0.9 & 1.5 & 1.75 & 1.85 & 1.95 & 2.25 & 2.4 \\
\hline $\begin{array}{c}\text { Small signal } \\
\text { gain (dB) }\end{array}$ & 36.4 & 35.8 & 35.3 & 34.3 & 34.1 & 33.4 & 32.0 & 30.8 & 30.8 \\
\hline Psat (dBm) & 33.9 & 34.7 & 34.5 & 35.1 & 34.6 & 33.6 & 33.8 & 33.3 & 33.5 \\
\hline $\begin{array}{c}\text { Maximum } \\
\text { PAE (\%) }\end{array}$ & 43 & 40 & 40 & 42 & 46 & 44 & 44 & 42 & 44 \\
\hline $\begin{array}{c}\text { Psat } \\
\text { in 50 MHz } \\
\text { (dBm) }\end{array}$ & 33.5 & 34.2 & 34.0 & 34.8 & 34.4 & 33.6 & 33.7 & 33.3 & 33.1 \\
\hline $\begin{array}{c}\text { Maximum } \\
\text { PAE } \\
\text { in 50 MHz } \\
(\%)\end{array}$ & 43 & 38 & 38 & 41 & 46 & 43 & 43 & 42 & 44 \\
\hline
\end{tabular}

each mode.

Figure 16 shows the frequency responses for the maximum Pout and PAE with W-CDMA (5-MHz adjacent chan-

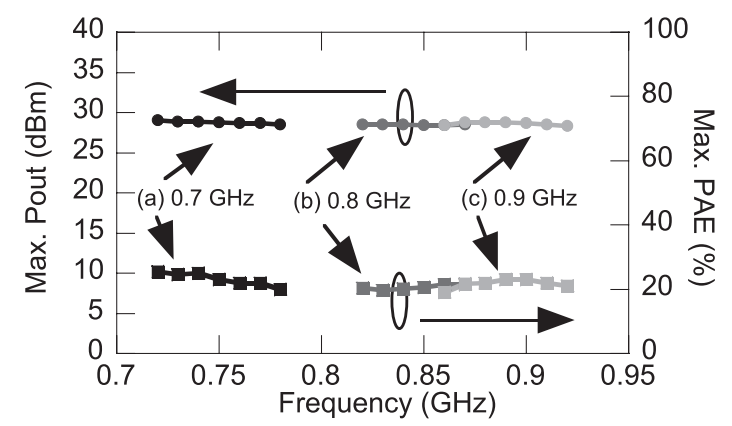

(a) Low band

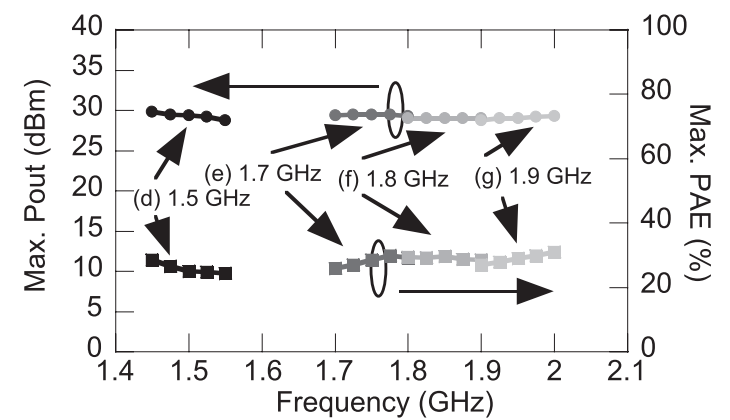

(b) Middle band

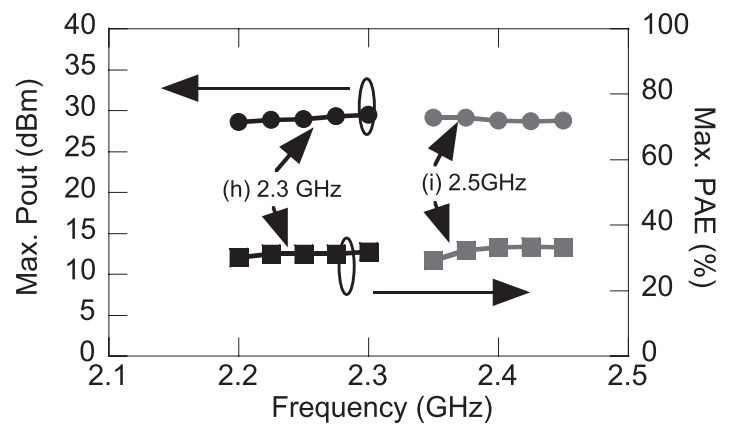

(c) High band

Fig. 16 Measured frequency responses of Pout and PAE for W-CDMA test signal with ACLR $<-37 \mathrm{dBc}$.

Table 4 Summary of Pout and PAE for W-CDMA test signal.

\begin{tabular}{|c|c|c|c|c|c|c|c|c|c|}
\hline Mode & (a) & (b) & (c) & (d) & (e) & (f) & (g) & (h) & (i) \\
\hline $\begin{array}{c}\text { Frequency } \\
\text { (GHz) }\end{array}$ & 0.75 & 0.85 & 0.9 & 1.5 & 1.75 & 1.85 & 1.95 & 2.25 & 2.4 \\
\hline $\begin{array}{c}\text { Pout } \\
(\mathrm{dBm})\end{array}$ & 28.8 & 28.5 & 28.7 & 29.4 & 29.5 & 29.0 & 29.0 & 29.0 & 28.8 \\
\hline PAE (\%) & 23 & 21 & 23 & 25 & 29 & 30 & 29 & 31 & 33 \\
\hline
\end{tabular}

nel leakage power ratio $(\mathrm{ACLR})<-37 \mathrm{dBc}$ ) for the (a) low band modes, (b) middle band modes, and (c) high band modes.

Table 4 shows a summary of the measurement results on the W-CDMA test with ACLR $<-37 \mathrm{dBc}$ test. The output power levels reach approximately $29 \mathrm{dBm}$ in all the bands. The maximum PAE values in each mode are 21 to $33 \%$. The PAE values are not so high. One of the reasons for the low PAE in the case of the W-CDMA test is that 
Table 5 Performance comparison of multi-band reconfigurable PA.

\begin{tabular}{|c|c|c|c|c|c|}
\hline Reference & $\begin{array}{c}\text { Frequency } \\
(\mathrm{GHz})\end{array}$ & $\begin{array}{c}\text { Gain } \\
(\mathrm{dB})\end{array}$ & $\begin{array}{c}\text { Pout } \\
(\mathrm{dBm})\end{array}$ & $\begin{array}{c}\text { PAE } \\
(\%)\end{array}$ & $\begin{array}{c}\text { Variable } \\
\text { Device }\end{array}$ \\
\hline$[9]$ & $0.9 / 1.8$ & $28-30$ & $30-31$ & 42 & PIN diode \\
\hline$[10]$ & $0.9 / 1.8 / 1.9 / 2.1$ & 13 & $27-28$ & $30-55$ & Varactor \\
\hline$[11]$ & $0.9 / 1.5 / 1.9$ & $9-14$ & $39-40$ & $61-65$ & PIN diode \\
\hline$[14]$ & $1 / 1.5 / 1.9 / 2.5$ & $10-12$ & $30-31$ & $40-55$ & MEMS \\
\hline This work & $\begin{array}{c}0.7 / 0.8 / 0.9 / 1.5 / 1.7 \\
11.8 / 1.9 / 2.3 / 2.4\end{array}$ & $30-36$ & $33-35$ & $40-46$ & $\begin{array}{c}\text { FET } \\
\text { switch }\end{array}$ \\
\hline
\end{tabular}

the PAE values are deteriorated by the difference in ACLR levels between the upper and lower sides due to a memory effect.

Table 5 summarizes the performance of the state-ofthe-art multi-band reconfigurable PA. A practical advantage of this work is that the proposed BS-MPA can operate in almost all the mobile communication bands with a moderate efficiency.

\section{Discussion}

This paper analyzes numerically the insertion loss of the MR-MN from the on-state switch. The numerical results show that it is preferable for the switch to have a low equivalent resistance in the on state such as the MEMS switch. For example, Table 6 shows the calculated results of the loss of the MR-MN employing one section, $L_{n 1}$, for matching in individual bands when $R_{\mathrm{sw}}=0.5 \Omega$, which is achieved by the MEMS switch employed in previous studies [14]-[16].

However, a comparison of " $L_{n 2}$ " in Table 2 and " $\mathrm{L}_{n 1}$ " in Table 6 shows that the proposed design scheme can decrease the loss from the semiconductor switches to the same level as that from the MEMS switch. On the other hand, insufficient isolation of the switch may produce a mismatch loss in the MR-MN. It is also possible to suppress the mismatching loss by designing the MR-MN by giving preliminary consideration to the isolation. In general, it is difficult for semiconductor switches to have a low insertion loss in the on-state and a high isolation in the off-state at the same time. Therefore, the MR-MN should employ switches with as a low equivalent resistance as possible, while maintaining the minimum isolation, which depends on the design of the MR-MN. The relationship between the isolation of the switch and insertion loss of the MR-MN is shown in [15].

In addition, as a requirement for the switch applied to the MR-MN, the voltage limitation of the switch is another point to be considered. Since the positions where the off-state switches are connected depends on the operating bands, it is useful to consider the maximum voltage that can be distributed on the TL. Figure 17 shows a circuit diagram for investigating the voltage on the TL of the MR-MN.

The RMS voltage, $V\left(d_{\mathrm{v}}\right)$, at distance of electrical length, $d_{\mathrm{v}}$, from the fixed MN on the TL can be written as

$$
V\left(d_{\mathrm{v}}\right)=\sqrt{P Z_{0} \frac{1+2\left|\Gamma_{\mathrm{g}}\right| \cos \left(2 d_{\mathrm{v}}-\theta_{\mathrm{g}}\right)+\left|\Gamma_{\mathrm{g}}\right|^{2}}{1-\left|\Gamma_{\mathrm{g}}\right|^{2}}} .
$$

Table 6 Calculation results on loss from MEMS switch

\begin{tabular}{|c|c|c|c|c|c|c|c|c|c|}
\hline $\begin{array}{c}\text { Frequency } \\
(\mathrm{GHz})\end{array}$ & 0.7 & 0.8 & 0.9 & 1.5 & 1.7 & 1.8 & 1.9 & 2.3 & 2.5 \\
\hline$L_{n 1}(\mathrm{~dB})$ & 0.72 & 0.64 & 0.58 & 0.32 & 0.22 & 0.17 & 0.14 & 0.02 & 0 \\
\hline
\end{tabular}

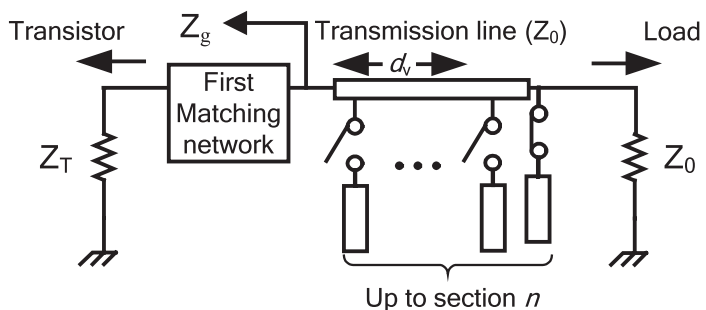

Fig. 17 Circuit diagram for investigating the voltage on the transmission line.

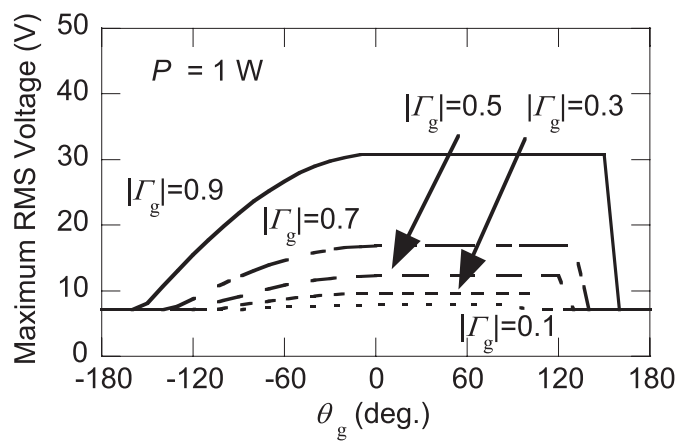

Fig. 18 Maximum root mean square RF voltage on the transmission line.

Then the maximum $V\left(d_{\mathrm{v}}\right)_{\max }$ on the TL depends on $\left|\Gamma_{\mathrm{g}}\right|$ and is given as

$$
V\left(d_{\mathrm{v}}\right)_{\max }=\left\{\begin{array}{cc}
\sqrt{P Z_{0}} \frac{1+2\left|\Gamma_{\mathrm{g}}\right| \cos \left(2 d_{\mathrm{v}}-\theta_{\mathrm{g}}\right)+\left|\Gamma_{\mathrm{g}}\right|^{2}}{1-\left|\Gamma_{\mathrm{g}}\right|^{2}} \\
\text { at } & \cos \left(\theta_{\mathrm{g}}\right)+\left|\Gamma_{\mathrm{g}}\right| \geq 0 \\
\text { at } & \cos \left(\theta_{\mathrm{g}}\right)+\left|\Gamma_{\mathrm{g}}\right| \geq 0
\end{array} .\right.
$$

Figure 18 shows plots of $V\left(d_{\mathrm{v}}\right)_{\max }$ as a function of $\theta_{\mathrm{g}}$. Here, parameters $P=1 \mathrm{~W}$ is selected as an example. The fixed $\mathrm{MN}$ should be designed considering the voltage limitation of the switch because $V\left(d_{\mathrm{v}}\right)_{\max }$ becomes large depending on $\left|\Gamma_{\mathrm{g}}\right|$, i.e., $V\left(d_{\mathrm{v}}\right)_{\max }$ is $30 \mathrm{~V}$ if $\left|\Gamma_{\mathrm{g}}\right|=0.9$ and $\theta_{\mathrm{g}}$ $=0$ degrees, as shown in Fig. 18 .

\section{Conclusion}

This paper presented a novel design scheme for a highly efficient BS-MPA employing the MR-MN. The design scheme focuses on reducing the power dissipation at the on-state switch in the MR-MN. The proposed scheme employs several sections that match the impedance in stages to minimize the power dissipation. The calculation results through derived equations on the loss of the MR-MN indicate quantitative improvement by applying the proposed scheme. According to a design example under practical conditions, 
the impedance transformation by the proposed scheme improves the insertion loss by more than $1 \mathrm{~dB}$. The fabricated LTCC 9-band BS-MPA including newly designed MR-MNs successfully switches its operating band from 0.7 to $2.4 \mathrm{GHz}$ through activation of the switches. A satisfactory output power level and PAE value in each band are also obtained in the CW test although the PAE at the input of a W-CDMA signal needs to be improved. The measurement results confirm that the proposed design scheme achieves low loss MRMN for a highly efficient BS-MPA. The BS-MPA will contribute greatly to achieving a compact multi-band mobile terminal. Future issues to be addressed are evaluating the loss of the MR-MN experimentally, achieving a BS-MPA with a more practical circuit size, and improving the PAE observed in the W-CDMA signal test after investigating in detail the distortion. As a practical BS-MPA, a compactsized BS-MPA is reported in [20] followed by the proposed scheme.

\section{References}

[1] G. Hueber and R.B. Staszewski, Multi-mode/Multi-band RF transceivers for wireless communications: Advanced techniques, Architectures, and Trends, Wiley-IEEE Press, 2011.

[2] A. Van Bezooijien, R. Mahmoudi, and A. Van Roermund, Adaptive RF front-ends for hand-held applications, Springer-Verlag, 2011.

[3] S. Zhang, J. Madic, P. Bretchko, J. Mokoro, R. Shumovich, and R. McMorrow, "A novel power-amplifier module for quad-band wireless handset applications," IEEE Trans. Microw. Theory Tech., vol.51, no.11, pp.2203-2210, Nov. 2003.

[4] W. Bakalski, B. Sogl, M. Zannoth, M. Asam, B. Kapfelsperger, J. Berkner, B. Eisener, W. Thomann, S. Marcon, W. Osterreicher, E. Napieralska, E. Rampf, A.L. Scholtz, and B-U. Klepser, "A quadband GSM/EDGE-Compliant SiGe-bipolar power amplifier," IEEE J. Solid-State Circuits, vol.43, no.9, pp.1920-1930, Sept. 2008.

[5] A. Sayed and G. Boeck, "Two-stage ultrawide-band 5-W power amplifier using SiC MESFET,' IEEE Trans. Microw. Theory Tech., vol.53, no.7, pp.2441-2449, July 2005.

[6] I.J. Bahl, "0.7-2.7-GHz 12-W power-amplifier MMIC developed using MLP technology," IEEE Trans. Microw. Theory Tech., vol.55, no.2, pp.222-229, Feb. 2007.

[7] S.H. Ji, C. Cho, J.W. Lee, and J. Kim, "Concurrent dual-band classE power amplifier using composite right/left-handed transmission lines," IEEE Trans. Microw. Theory Tech., vol.55, no.6, pp.13411347, June 2007

[8] P. Colantonio, F. Giannini, R. Giofre, and L. Piazzon, "A design technique for concurrent dual-band harmonic tuned power amplifier," IEEE Trans. Microw. Theory Tech., vol.56, no.11, pp.25352555, Nov. 2008.

[9] D.E. Dawson, "Closed-form solutions for the design of optimum matching networks," IEEE Trans. Microw. Theory Tech., vol.57, no.1, pp.121-129, Jan. 2009.

[10] Y. Lu, D. Peroulis, S. Mohammadi, and L.P.B. Katehi, "A MEMS reconfigurable matching network for a class $\mathrm{AB}$ amplifier," IEEE Microw. Wireless Compon. Lett., vol.13, no.10, pp.437-439, Oct. 2003.

[11] H. Zhang, H. Gao, and G-P. Li, "Broad-band power amplifier with a novel tunable output matching network," IEEE Trans. Microw. Theory Tech., vol.53, no.11, pp.3606-3614, Nov. 2005.

[12] W.C.E. Neo, Y. Lin, X. Liu, L.C. N. de Vreede, L.E. Larson, M. Spirito, M.J. Pelk, K. Buisman, A. Akhnoukh, A. de Graauw, and L.K. Nanver, "Adaptive multi-band multi-mode power amplifier using integrated varactor-based tunable matching networks," IEEE J. Solid-State Circuits, vol.41, no.9, pp.2166-2176, Sept. 2006.
[13] H.M. Nemati, J. Grahn, and C. Fager, "Band-reconfigurable LDMOS power amplifier," Proc. 40th European Microwave Conference, pp.978-981, Paris, 2010.

[14] A. Fukuda, H. Okazaki, T. Hirota, and Y. Yamao, "Novel $900 \mathrm{MHz} / 1.9 \mathrm{GHz}$ dual-mode power amplifier employing MEMS switches for optimum matching," IEEE Microw. Wireless Compon. Lett., vol.14, no.3, pp.121-123, March 2004.

[15] A. Fukuda, H. Okazaki, T. Hirota, and Y. Yamao, "Novel band-reconfigurable high efficiency power amplifier employing RF-MEMS switches," IEICE Trans. Electron., vol.E88-C, no.11, pp.2141-2149, Nov. 2005.

[16] A. Fukuda, H. Okazaki, and S. Narahashi, "Highly efficient multiband power amplifier employing reconfigurable matching and biasing networks," IEICE Trans. Electron., vol.E93-C, no.7, pp.949957, July 2010.

[17] A. Fukuda, K. Kawai, T. Furuta, H. Okazaki, S. Oka, S. Narahashi, and A. Murase, "A high power and highly efficient multi-band power amplifier for mobile terminals," Proc. Radio and Wireless Symposium (RWS), pp.45-48, New Orleans, 2010.

[18] T. Furuta, A. Fukuda, K. Kawai, H. Okazaki, and S. Narahashi, "Multi-band power amplifier on an LTCC Substrate," IEEE Topical Symposium on Power Amplifiers for Wireless Communications (PAS), Sept. 2010.

[19] D.M. Pozar, "Impedance matching and tuning," in Microwave Engineering, Chapter 5, pp.251-299, John Wiley \& Sons, 1998.

[20] T. Furuta, A. Fukuda, K. Kawai, H. Okazaki, and S. Narahashi, "Compact $1.5 \mathrm{GHz}$ to $2.5 \mathrm{GHz}$ multi-band multi-mode power amplifier,” IEICE ELEX, June 2011.

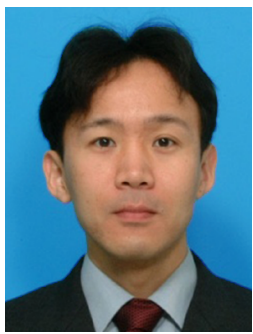

Atsushi Fukuda received the B.E. and M.E degrees in electrical engineering from Tokyo University of Science, Tokyo, Japan, in 1997 and 1999, respectively. In 1999, he joined NTT DOCOMO, INC., where he is involved in research on RF circuits and development on base station equipments for mobile communication systems. He is a member of the IEEE.

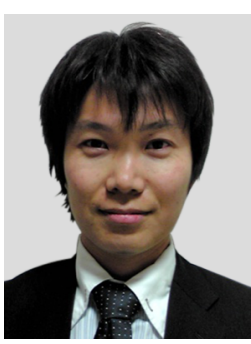

Takayuki Furuta

received the B.E. and M.E. degrees in electric engineering from Hokkaido University, Sapporo, Japan, in 2002 and 2004, respectively. In 2004, he joined NTT DoCoMo wireless laboratories, where he is involved in research on RF and microwave circuits for mobile communication systems. Mr. Furuta was the recipient of the 2010 Young Researcher's Award presented by IEICE. 


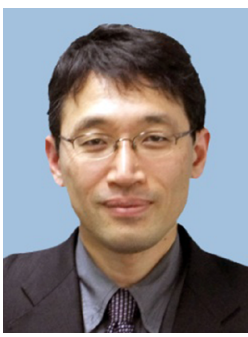

Hiroshi Okazaki received the B.E. and M.E. degrees from Osaka University, Osaka, Japan, in 1988 and 1990, respectively, and Dr. Eng. degree from Tokyo Institute of Technology, Tokyo, Japan, in 2011. In 1990, he joined NTT Radio Communication Laboratories, Kanagawa, Japan, where he was engaged in research on microwave circuits and equipments for communication systems. During 20012003, he was with NTT Electronics Corporation, Tokyo, Japan, where he was engaged in development of ultra-high-speed devices for photonic communication systems. Since 2003, he has been with NTT DOCOMO, INC., where he is involved in research on RF circuits for mobile communication systems. Dr. Okazaki was the recipient of the 1997 Young Engineer Award presented by IEICE and the 1998 Japan Microwave Prize at 1998 Asia Pacific Microwave Conference. He is a senior member of the IEEE.

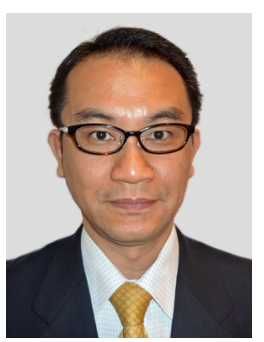

Shoichi Narahashi received the B.E. and M.E. degrees from Kumamoto University, Kumamoto, Japan, in 1986 and 1988, respectively, and the Ph.D. degree from Hokkaido University, Sapporo, Japan, in 2008. In 1988, he joined Nippon Telegraph and Telephone (NTT) Radio Communication Systems Laboratories where he was engaged in research and development on base station equipment for digital mobile communications. Since 1992, he has been with NTT DOCOMO, INC. He is currently an Executive Research Engineer of Research Laboratories with the mission of investigating RF circuits for mobile communications. He is a member of the IEEE and the Society of Instrument and Control Engineers (SICE).

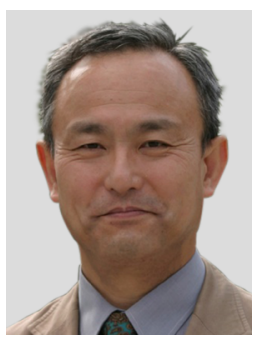

Toshio Nojima received his M.E. and $\mathrm{Ph} . \mathrm{D}$. degrees in electronic engineering from Hokkaido University, Sapporo, Japan. From 1974 to 1992, he was with Nippon Telegraph and Telephone (NTT) Communications Laboratories, where he was engaged in the research and development of high capacity microwave relay systems (SSB-AM and 256QAM systems). From 1992 to 2001, he was with NTT DoCoMo, Inc., where he was a Senior Executive Research Engineer and pursued researches on the mobile communication RF technologies including EMC. Currently, he is a Professor of the graduate school of Information Science and Technology, Hokkaido University. He is a member of the IEEE and the Bioelectromagnetics Society (BEMS). 